\title{
Window Function Analysis of Nonlinear Behaviour of Fourth Fundamental Passive Circuit Element: Memristor
}

\author{
Raj Kumar Singh ${ }^{1 *}$ \\ 1* Department of Physics, RLSY College, Ranchi University, Ranchi, India
}

\begin{abstract}
Memristor, first time proposed by Prof. Leon O. Chua in 1971, is the 'missing' fourth fundamental passive circuit element which has a memory retention capacity but of course they can't store energy. Memristor model realised for the first time in HP laboratory in 2008 was based on linear ion drift model. Though it worked well along the length of device, there is no explanation on the behaviour of device at the boundary. Various window functions have been introduced with their own merits and limitations to address this problem.
\end{abstract}

Key words: memristor, memory, model, window function.

\section{Introduction}

In 1971, Leon Chua [1] postulated the existence of the fourth 'missing' fundamental circuit element, called the memristor. A memristor could be charge-controlled or flux-controlled. It is said to be chargecontrolled if the relation between flux and charge is expressed as a function of electric charge and is said to be flux-controlled if the relation between flux and charge is expressed as a function of the flux linkage [2-6]. Particularly, for a charge-controlled memristor,

$$
\phi=f(q)
$$

Which upon differentiation yields

$$
\frac{d \phi}{d t}=\frac{d f(q)}{d q} \times \frac{d q}{d t}
$$

or,

$$
v(t)=M(q) i(t)
$$

where,

$$
v(t)=d \phi / d t \text { is the voltage }
$$

and

$$
M(q)=d f(q) / d q
$$

$M(q)$ is called as memristance

When the charge flows in one direction through the circuit, resistance of the memristor increases, and its resistance decreases when the charge flows in the opposite direction. If the applied voltage is turned off, the memristor "remembers" the last resistance it had. When the flow of charge is started again, the resistance of the circuit will be what it was when it was last active. A memristive system [7] behaves as a linear resistor in the limit of infinite frequency and as a non-linear resistor in the limit of zero frequency. Appearance of a "pinched hysteretic loop" (fig. 1) in the current-voltage characteristics of memristor, when subject to a periodic input voltages [6], is a noticeable property of it.

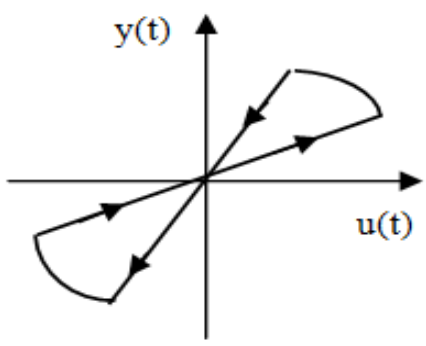

Fig. 1: Pinched hysteresis loop for the generalized response functions. 
Memristive behavior has been observed in several systems viz thermistors [9], molecular systems [10], spin electronic devices [11] and nanostructures due to thin films [7-14], as well as various examples mentioned due to Chua. Rest of the paper is organized as follows. In Section 2 we describe the HP lab Linear Ion Drift model of memristor. Section 3 describes different types of popular window functions with their merits and limitations. At the end we conclude in Section 4.

\section{Hp Memristor: Linear Ion Drift Model}

HP lab linear ion drift model of memristor is based on the physical structure of the device. It is assumed that the width $D$ of the device has two regions. One region is doped with positive oxygen ions and thus has oxygen vacancies and the other side is undoped. The doped region of width $w$ (which acts as the state variable), conducts more and hence has lower resistance than the undoped region which has high resistance because it doesn't conduct until oxygen vacancies from the doped region penetrate into it. It is assumed that the ion drift is linear and the ions have equal average ion mobility $\mu_{\mathrm{v}}$. Figure 2 below shows the model of the HP memristor.

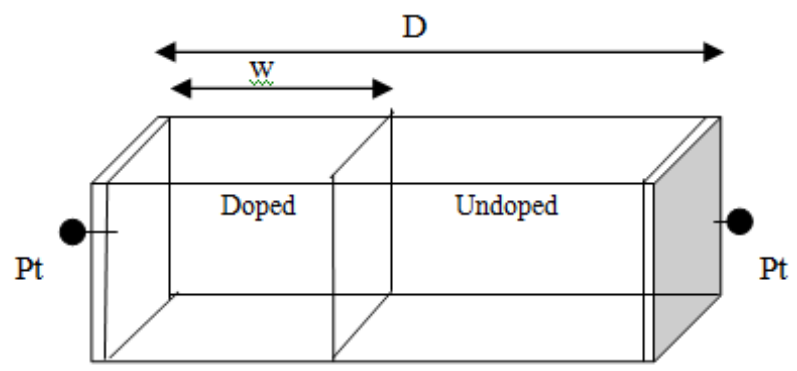

Fig. 2: Schematic of HP Memristor.

The state equation can be written as after [9], voltage across the terminals of memristor

$v(t)=\left[R_{o n} x(t)+R_{o f f}(1-x(t))\right] i(t)$

Where $\quad x(t)=\frac{w(t)}{D}$ is the normalized state variable, $\mathrm{w}$ being the thickness of doped region and $\mathrm{D}$ the thickness of the undoped region. $\mathrm{R}_{\mathrm{on}}$ and $\mathrm{R}_{\mathrm{off}}$ are the $\mathrm{ON}$ and $\mathrm{OFF}$ state resistance of memristor. whence

$M_{0}=\left[R_{o n} x\left(t_{0}\right)+R_{o f f}\left(1-x\left(t_{0}\right)\right)\right]$

With $\mathrm{M}_{0}$ being the the memristance at $\mathrm{t}=0$.

$M_{0}=R_{o n}\left[x\left(t_{0}\right)+r\left(1-x\left(t_{0}\right)\right)\right]$

With $r$ being the ratio of $R_{\text {on }}$ and $R_{\text {off }}$

The current voltage relation for the memristor leads to

$$
i(t)=\frac{v(t)}{R_{o f f}\left(\sqrt{1-\frac{2 \mu_{D}}{r D} \phi(t)}\right)}
$$

\section{Window Functions}

Window function is the additional term in the state equation of memristor which models the nonlinear behaviour at the device boundary. Some of the window functions in use for the linear ion drift model are discussed hereafter. 


\subsection{STRUKOV ET AL WINDOW FUNCTION}

As discussed, the boundary value restrictions can be modelled using a window function.

$$
\begin{aligned}
\mathrm{V}_{\mathrm{D}} & =\mathrm{dw} / \mathrm{dt} \\
& =\eta \mu_{\mathrm{D}} \mathrm{R}_{\mathrm{ON}} / \mathrm{D} * \mathrm{I}(\mathrm{t}) \cdot \mathrm{F}(\mathrm{x})
\end{aligned}
$$

where $V_{D}$ is the boundary drift velocity, and $x=w / D$ is the normalized state variable. $F(x)$ should reach its maximum in the centre of the device, $\mathrm{x}=0.5$, and decrease toward boundaries in which it will reach zero speed at the terminal states $\mathrm{x}=0$ or $\mathrm{x}=1$. This was exactly what Strukov et al. [7] proposed as their widow function (Figure 3):

$\mathrm{F}(\mathrm{w})=\mathrm{w}(1-\mathrm{w}) / \mathrm{D}^{2}$

Subject to the boundary conditions as

$\mathrm{F}(0)=0$

$\mathrm{F}(\mathrm{D}) \simeq 0$.

This function supports the essential boundary conditions, but the inherent problem with this window function is that at the terminal states when

$\mathrm{w} \rightarrow 0, \mathrm{dw} / \mathrm{dt}=0$

and $\mathrm{w} \rightarrow \mathrm{D}, \mathrm{dw} / \mathrm{dt}=0$

This means that no external field can change the state at the boundary and this is a great concern. Another fundamental problem due to the assumptions made is that memristor remembers the amount of charge passing through the device, but it appears that, it remembers the position of the state boundary between doped and undoped regions.

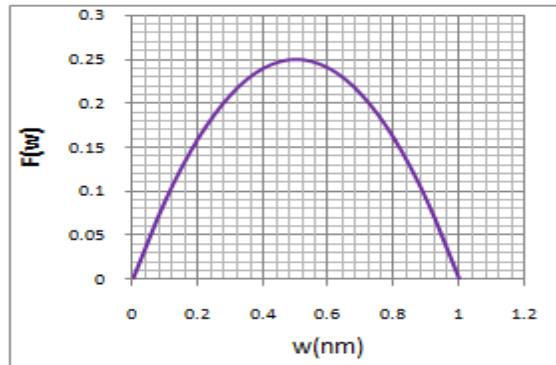

Fig. 3: Strukov et al Window Function.

\subsection{BENDERLI AND WEY WINDOW FUNCTION}

With slight variation with Strukov et al, Benderli \& Wey [15] proposed another window function in 2009 by just replacing (1-w) by (D-w):

$\mathrm{F}(\mathrm{w})=\mathrm{w}(\mathrm{D}-\mathrm{w}) / \mathrm{D}^{2}$

With clear boundary conditions:

$\mathrm{W} \rightarrow 0, \quad \mathrm{~F}(\mathrm{w}) \rightarrow 0$

$\mathrm{W} \rightarrow \mathrm{D}, \mathrm{F}(\mathrm{w}) \rightarrow 0$

This could meet both boundary conditions. As it can be seen, the above functions can approximate the nonlinear behaviour of when the memristor is not in the boundary states.

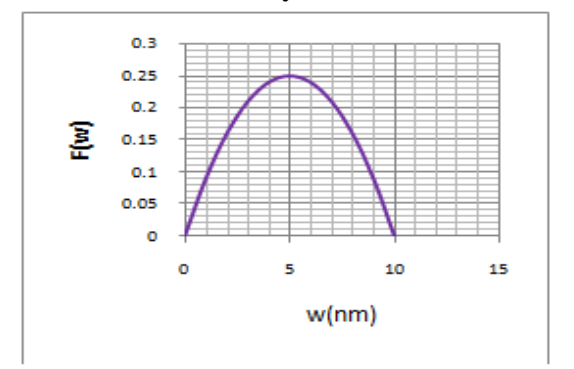

Fig. 4: Benderli and Wey Window Function. 
Benderli and Wey also assumed in their window function that memristor remembers the amount of charge passing through the device, this is not the case as in Strukov et al. Rather it is said that, it remembers the position of the state boundary between the two regions.

\subsection{JOGLEKAR WINDOW FUNCTION}

Joglekar and Wolf proposed in 2009 [16], to address the problems with the Strukov et al and Benderli \& Wey's window function, window function by introducing a control parameter, (p) in it.

$\mathrm{F}(\mathrm{x})=1-(2 \mathrm{x}-1)^{2 \mathrm{p}}$

in which $\mathrm{p}$ is the control parameter and $\mathrm{x}=\mathrm{w} / \mathrm{D}$. Both $\mathrm{p}$ and $\mathrm{x}$ are positive integers. The function looks similar to the rectangular window function when the value of $\mathrm{p}$ grows beyond 2 (Figure 5), and the nonlinear drift phenomenon decreases. The boundary conditions are simple as

$\mathrm{F}(0)=0$ and $\mathrm{F}(1)=0$.

Some of the Joglekar window function polynomials are tabulated below.

TABLE 1 Joglekar window function polynomials for different value of control parameter

\begin{tabular}{|l|l|}
\hline$p$ & \multicolumn{1}{|c|}{ Joglekar and Wolf function $F(x)$} \\
\hline 1 & $4 x(1-x)$ \\
\hline 2 & $-16 x^{4}+32 x^{3}-24 x^{2}+8 x$ \\
\hline 3 & $-64 x^{6}-192 x^{5}-240 x^{4}+160 x^{3}-60 x^{2}+12 x$ \\
\hline 4 & $-256 x^{8}+1024 x^{7}-1792 x^{6}+1792 x^{5}-1120 x^{4}+448 x^{3}-112 x^{2}+16 x$ \\
\hline 5 & $-1024 x^{10}+5120 x^{9}-11520 x^{8}+15360 x^{7}-13440 x^{6}+8064 x^{5}-3360 x^{4}+960 x^{3}-180 x^{2}+20 x$ \\
\hline 6 & $-4096 x^{12}+24576 x^{11}-67584 x^{10}+112640 x^{9}-12672 x^{8}+101376 x^{7}-59136 x^{6}+25344 x^{5}-7920 x^{4}+1760 x^{3}-264 x^{2}+24 x$ \\
\hline
\end{tabular}
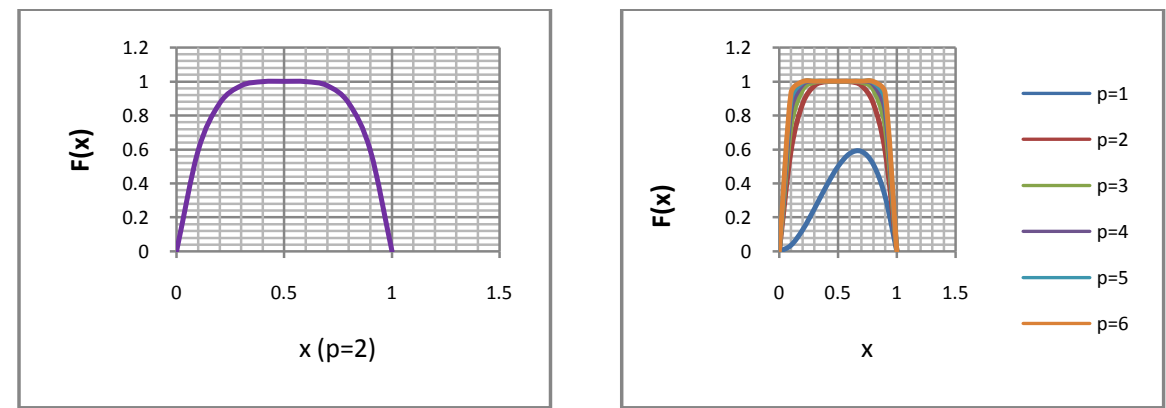

Fig. 5: Joglekar and Wolf Window Function.

The modified state change equation:

$\mathrm{dw} / \mathrm{dt}=\eta \mu_{\mathrm{D}} \mathrm{R}_{\mathrm{ON}} / \mathrm{DI}(\mathrm{t})\left[1-(2 \mathrm{x}-1)^{2 \mathrm{p}}\right]$

At the boundaries, it was noticed that the state variable in some sense, stuck at the boundary states, and it is difficult to be changed. To be more specific, no external stimulus at terminals can change the state variable.

\subsection{BIOLEK WINDOW FUNCTION}

Biolek et al [17] came up with a solution to address inaccuracy of Joglekar's window function. They presented another window function that depends on memristor current $i$ in addition to normalized state variable $x$ and the control parameter $p$ :

$F(x)=1-(x-\operatorname{sgn}(-i))^{2 p}$

As suggested by Biolek et al, $\operatorname{sgn}(-i)=1$ when $i \geq 0$ (current is positive for the expanding doped region) and $\operatorname{sgn}(-i)=0$ when $i<0$. The problem with Biolek's window function is that there is no continuity condition at the boundaries.

First three Biolek window function polynomials are tabulated below in table 2 . 
TABLE 2 Biolek window function polynomials for different values of control parameter

\begin{tabular}{|c|c|c|c|}
\hline \multicolumn{2}{|c|}{ Sgn(-i) $=1$} & \multicolumn{2}{c|}{ Sgn(-i) $=0$} \\
\hline $\mathrm{P}$ & Biolek function F(x) & $\mathrm{p}$ & Biolek function F(x) \\
\hline 1 & $-\mathrm{x}^{2}+2 \mathrm{x}$ & 1 & $1-\mathrm{x}^{2}$ \\
\hline 2 & $-\mathrm{x}^{4}+4 \mathrm{x}^{3}-6 \mathrm{x}^{2}+4 \mathrm{x}$ & 2 & $1-\mathrm{x}^{4}$ \\
\hline 3 & $-\mathrm{x}^{6}+6 \mathrm{x}^{5}-15 \mathrm{x}^{4}+20 \mathrm{x}^{3}-15 \mathrm{x}^{2}+6 \mathrm{x}$ & 3 & $1-\mathrm{x}^{6}$ \\
\hline
\end{tabular}
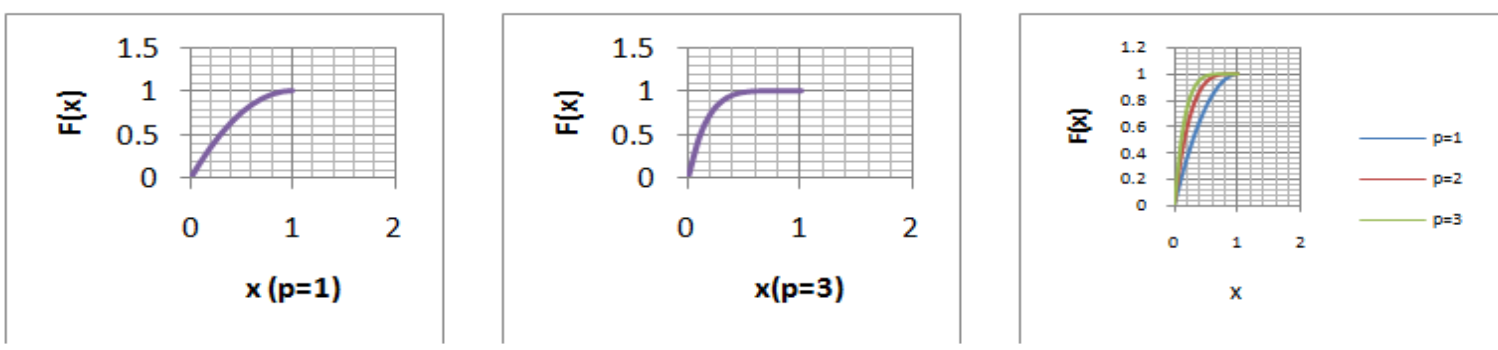

Fig. 6: Biolek window function for $\operatorname{sgn}(-i)=1$.
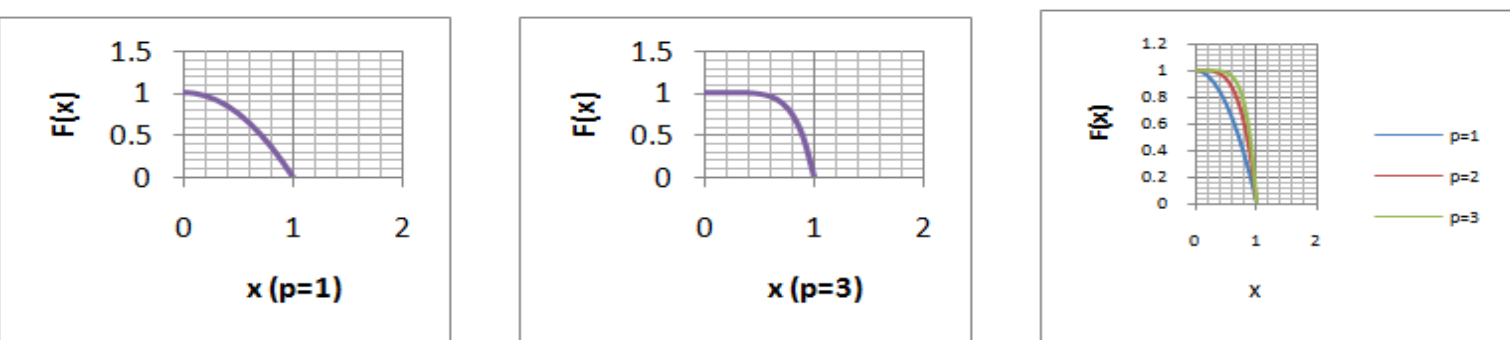

Fig. 7: Biolek window function for $\operatorname{sgn}(-i)=0$.

Joglekar's and Biolek's window functions meet the boundary conditions but Biolek's window function fails to achieve the continuity condition at the boundaries.

\subsection{PRODROMAKIS WINDOW FUNCTION}

One problem with the window functions is that the maximum value of the function is only one and cannot be lower or higher than that. This issue has been resolved by Prodromakis et al. through their new window function with two control parameters $j$ and $p[18]$ :

$\mathrm{F}(\mathrm{w})=\mathrm{j}\left[1-\left\{(\mathrm{w}-1 / 2)^{2}+3 / 4\right\}^{\mathrm{p}}\right]$

where $j$ is a control parameter that specifies the highest value of the function,

which can be lower or higher than one. Prodromakis window function can be understood in two ways: first $j$ is assumed constant and $p$ varying and in the second form $p$ is taken constant and $j$ varied. A few of the Prodromakis functions are tabulated below in table 3 .

TABLE 3 Prodromakis function under different control conditions

\begin{tabular}{|c|c|c|c|}
\hline \multicolumn{2}{|c|}{$\mathrm{j}=1$ (constant), $\mathrm{p}$ varying } & $\mathrm{j}$ & $\mathrm{j}=1$ (constant), $\mathrm{j}$ varying \\
\hline $\mathrm{p}$ & Prodromakis function $\mathrm{F}(\mathrm{x})$ & Prodromakis function $\mathrm{F}(\mathrm{x})$ \\
\hline 1 & $-\mathrm{x}^{2}+\mathrm{x}$ & $\mathrm{j}=0.2,0.4,0.6,0.8$, & $\mathrm{j}\left(-\mathrm{x}^{2}+\mathrm{x}\right)$ \\
\hline 2 & $-\mathrm{x}^{4}+2 \mathrm{x}^{3}-3 \mathrm{x}^{2}+2 \mathrm{x}$ & 1.0 & \\
\hline
\end{tabular}
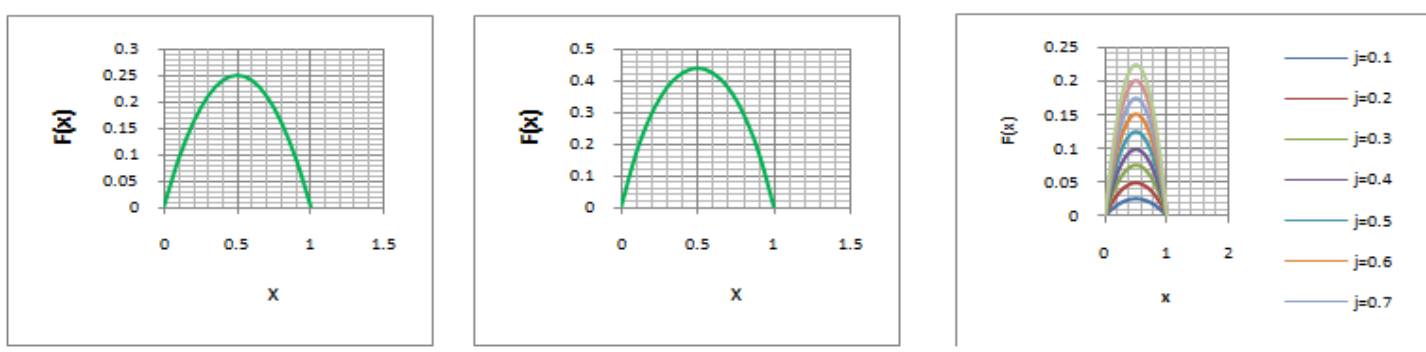

Fig. 8: Prodromakis window function, $j=1$ (constant) and $p$ varying (first two graphs from left), and Prodromakis window function, $p=l$ (constant) and $\mathrm{j}$ varying (right side graph). 


\section{Conclusion}

An analysis of various window functions for the linear ion drift model of memristor has been discussed. Each window function attempts to address the nonlinear relationship between current and voltage at the boundaries of the HP memristor model. While Strukov et al and Benderli \& Wey window function failed to maintain the continuity condition at the boundary, however they succeed in meeting the essential boundary condition. Joglekar \& Wolf and Biolek introduced control parameter in their window function and thus succeed in explaining the nonlinearity at the boundary couldn't offer any explanation to the question that when memristor is at the terminal states, no external excitation can change its state. All window functions assume that the memristor remembers the amount of charge that flows through it, but it appears that memristor remembers the position of boundary between the doped and undoped regions. Thus future works may address this problem of handling nonlinearity in the window function possibly by assuming that the memristors remember the position of boundary between the two regions which achieve the changes in resistance due to some well explained reasons.

\section{References}

[1]. Chua, L.O., IEEE Trans. Circuit Theory, "Memristor- the missing circuit element”, 1971;18(5)5:507-519. 1971, vol. CT-18, no. 5, Pp 507-519.

[2]. Pershin, Y. V. and M. Di Ventra, 2009, "Putting memory into circuit elements: memristors, memcapacitors and meminductors", Phys. Rev. B 79(15), 153307

[3]. Singh, Raj Kumar and Mamta, Kumari, "Memcapacitor and memcapacitive systems: Basic fingerprints of nanoscale memory", African Journal of Science and Research, 2014, (3) 4:16-20.

[4]. Singh, Raj Kumar and Mamta, Kumari, "Spin-based memory: Memristive devices and their applications", European Scientific Journal, Vol. 10, No. 18, Pp 230-246, June 2014

[5]. S. Mohanty, "Memristors: From basics to Deployment", IEEE Potentials, 0278-6648/May-June 2013@2013IEEE

[6]. C. H. Mejia, A. S. Reyes and H. V. Leal, "A Family of Memristive Transfer Functions of Negative Feedback Nullor-based Amplifiers", IEEE, 978-1-4673-4900-0/2013.

[7]. D. B. Strukov, G. S. Snider, D. R. Stewart and R. S. Williams, "The missing memristor found", Nature, 453 (7191), 80-83, 2008.

[8]. Chua, L.O. and Kang, "Memristive devices and systems", S.M, Proceedings of the IEEE, 1976, vol. 64, no. 2, Pp 209-223

[9]. M. Sapoff and R. M. Oppenheim, "Theory and application of self-heated thermistors", Proc. IEEE, vol. 51, pp. 1292-1305, 1963

[10]. Y. Chen, G. Y. Jung, D. A. A. Ohlberg, X. M. Li, D. R. Stewart, J. O. Jeppesen, "Nanoscale molecular-switch crossbar circuits", Nanotechology. 2008; 14:462- 468.

[11]. Di Ventra, M, "Electrical transport in nanoscale system", Cambridge University Press, 2008.

[12]. J. J. Yang, M. D. Pickett, X. Li, D. A. A. Ohlberg, D. R. Stewart, and R. S. Williams, "Memristive switching mechanism for metal/oxide/metal nanodevices", Nature Nanotechnology, vol. 3, pp. 429-433, 2008.

[13]. T Driscoll, H.T. Kim, B.G. Chae, M. Di Ventra, and D. N. Basov, "Memristive Adaptive filters", arXiv:0901.0899, 2009.

[14]. J. Blanc and D. L. Staebler, "Electrocoloration in SrTio3: Vacancy drift and oxidation-reduction of transition metal", Phys. Rev. B, 4,100: 3548-3557, 1971

[15]. T. A. Wey and S. Benderli, "Amplitude modulator circuit featuring $\mathrm{TiO}_{2}$ memristor with linear dopant drift", Electronics Letters, vol. 45, no. 22, pp. 1103-1104, 2009.

[16]. Y. N. Joglekar and S. J. Wolf, "The elusive memristor: properties of basic electrical circuits," European Journal of Physics, vol. 30, pp. 661-683, 2009

[17]. Z. Biolek, D. Biolek, and V. Biolkova, "Spice model of memristor with nonlinear dopant drift," Radioengineering, vol. 18, no. 2, pp. 210-214, 2009.

[18]. T. Prodromakis, B. P. Peh, C. Papavassiliou, and C. Toumazou, "A Versatile Memristor Model With Nonlinear Dopant Kinetics," IEEE Transactions on Electron Devices, vol. 58, no. 9, pp. 3099-3105, 2011. 